\title{
Ear Recognition by using Least Mean Square Method
}

\author{
Sanjiv K. Mishra \\ Department of Information \\ Technology \\ RKDF(RGPV),Bhopal, M.P \\ India
}

\author{
Shrikant Lade \\ Department of Information \\ Technology \\ RKDF(RGPV),Bhopal, M.P \\ India
}

\author{
Manish K. Suman \\ Department of Information \\ Technology \\ RKDF(RGPV),Bhopal, M.P \\ India
}

\begin{abstract}
Biometrics is a proper way of identifying human beings based on certain physiological characteristics and behavioral characteristics. Some of the physiological characteristics are fingerprint, face, DNA and iris. Some of the useful behavioral traits are gait and voice. But ear biometrics has recently emerged as a new area in biometrics. A great possibility for use of human ears for identification exists but no automated ear biometrics system has been used yet. Automatic analysis in biometrics reduces time and helps us to accomplish tasks in a quicker and efficient manner. In the present paper, an ear recognition based on least mean square method is proposed. That is the mean square method is based on the approximate curve between the intensity and distance from the center of the ear. So overall work is broadly divided in to three parts. First segment the ear i.e. ROI(region of interest). Second apply the method of least square and finally identify the ear by minimum average distance matching. Experimental results show that the proposed algorithm achieve better result from other 2D ear recognition techniques.
\end{abstract}

\section{General Terms}

Biometrics , physiological characteristics , behavioral characteristics, ears.

\section{Keywords}

Ear recognition, least square method, segmentation, approximate curve, automated ear biometrics, distance matching.

\section{INTRODUCTION}

Automatically recognizing a person by certain physiological characteristics like fingerprint, face, iris , ear, hand geometry, DNA or behavioral characteristics like gait, voice, signature of the person. Automating biometrics is a challenging problem in the field of image processing and pattern recognition. Ear biometrics has come as a new biometrics field. Ears because of their stability, very less variability by age and easy availability [1] can be used for biometrics.

Techniques used currently for personal authentication can be broadly divided into two categories, in one category a person has to carry some tokens like personal identity cards. In other method instead of carrying object, person carries some information like personal identification number or password. A mix of these two ways is generally used for authentication of a person. Carrying objects has problems associating with them like the person forget to carry object with him, object getting lost and theft . On the other hand user may forget his/her user name/password. In addition, hackers can guess passwords for unethical reasons. Biometrics is a domain that aims to give the best answer to these problems. The word 'biometrics' is derived from two Greek words - bios and metric Biometrics simply means life measurement. As we know that biometrics characteristics is personally attached to the person to be authenticated. That is biometric measures are unique for each person. For example, the fingerprint is unique. There are also many applications of biometrics. Some of them are as follows :

(1) Biometrics-based attendance system

(2) Biometrics-based authentication system

(3) Safes and locks

(4) Forensic application

(5) Security devices

Because of the above advantages, biometrics is emerging as new tool for personal authentication.

\section{LITERATURE REVIEW}

Many techniques for identifying a person using his ears have been proposed. The method implemented by Iannarelli is probably the first where over 10000 ears are examined for their uniqueness [2]. However, the experiments are conducted manually without automating the methods to extract the specific key points. Burge and Burger's work [3] where they have proposed an error correcting graph matching of adjacency graphs formed from generalized Voronoi diagram formed from ear edge curves. But the edges detected from the ear have shown considerable variation even in presence of relatively small changes in camera-to-ear orientation or lighting.Hurley et al. [4] introduced a method based on energy feature of the image. But results show that feature vector is highly immune to initialization, rotation, scale and noise.Victor et al. [5] gave Eigen ear using PCA based feature extraction. Their work gives the reliability of the ear images for personal authentication. But their work was not fully automated. Moreno et al. [6] have given a multiple identification method. They have used different classifiers. Among feature extraction methods one is based on computing ear feature points heuristically, other one considers morphology of outer ear image, in one case compression network was trained. Finally various classification techniques are used like voting, Bayesian combinations linear weighing and neural networks. The best identification rate of $93 \%$ is achieved using compression networks. Hurley et al. [7] presented a novel approach to ear identification called the force field transform. They achieved a rank- one recognition rate of $99.2 \%$ (compared to $62.4 \%$ for PCA) on the XM2VTS dataset, but on a limited probe dataset of 63 subjects.

Bustard \& Nixon , 2010 [8] have recently exploited the scale invariant feature transform (SIFT) for matching ear images and illustrated promising result. Abate et al., 2006 
[9] presented a spectral feature for the characterization of ear images. Chang et al., 2003 [10] , Moreno et al., 1999 [11] , Mu et al., 2004 [12] , Hurley et al., 2002 [13] , Hurley et al., 2005 [14] , Choras , 2004 [15] , AbdelMottaleb et al., 2006 [16] , Arbab-Zavar \& Nixon , 2007 [17] presented a structural features for the characterization of ear images. Chen \& Bhanu , 2007 [18] , Bhanu \& Chen , 2003 [19] , Theoharis et al., 2008 [20] , Yan \& Bowyer, 2007 [21] , presented a 3-D features for the characterization of ear images. However such approaches have only been tested on small scale databases and in most cases the size of the probe dataset has been less than 100 subjects and on the other hand these uses 3D scanners, which are very expensive. Moreover , 3D image algorithms are not much compatible with forensic science or police procedures where $2 \mathrm{D}$ imaging is more relevant. Therefore our focus in this work has been to exploit the 2D ear images that can be conveniently acquired from conventional digital camera.

\section{PROPOSED WORK}

The approach for ear detection from side face image can be divided into few steps. Firstly ear segmentation is performed and then approximate the curve between intensity values and distance from the center of the ear. For approximation we use the method of least square. Now finally we use matching technique and identify the ear.

\subsection{Block Diagram}

First show the block diagram of the process for ear recognition(see Figure 3.1)

$$
d_{\text {final }}=\sum_{i=1}^{n}\left[y_{i}-\left(m x_{i}+c\right)\right]^{2}
$$

For the best fit line with least value of error, $m$ and $c$ are given by

$$
\begin{aligned}
m & =\frac{n \sum_{i=1}^{n} i y_{i}+\sum_{i=1}^{n} i \sum_{i=1}^{n} y_{i}}{n \sum_{i=1}^{n} i^{2}-\left(\sum_{i=1}^{n} i\right)^{2}} \\
c & =\frac{\sum_{i=1}^{n} i y_{i}+m \sum_{i=1}^{n} i}{n}
\end{aligned}
$$

\subsection{Algorithm}

Step1: Start

Step 2: Approximate the curve between intensity vs. distance with a line.

Step 3: For approximation

For $\mathrm{i}=1$ to $\mathrm{n}$

For $\mathrm{j}=\mathrm{i}$ to $\mathrm{n}$

Calculate e[1,i,j] using method of least squares.

While (e $[\mathrm{k}, 1, \mathrm{n}]>\mathrm{n} *$ maxerror) For $\mathrm{i}=1$ to $\mathrm{n}$

For $\mathrm{j}=1$ to $n$

$\mathrm{e}[\mathrm{k}+1, \mathrm{i}, \mathrm{j}]=\operatorname{Min}(\mathrm{e}[\mathrm{k}, \mathrm{i}, \mathrm{s}]+\mathrm{e}[1, \mathrm{~s}+1, \mathrm{j}])$ where $\mathrm{i}<\mathrm{s}<\mathrm{j}$

Step 4: Find the average value of the distance of all the concurrent lines of the two ears.

Step 5: average value gives the matching score.

Step 6: End

Now implement the algorithm for this:

First take the segmented ear image with 20 concurrent lines passing through center of ear, intensity variation along whom is taken for matching the ears (see Figure 3.4.1)

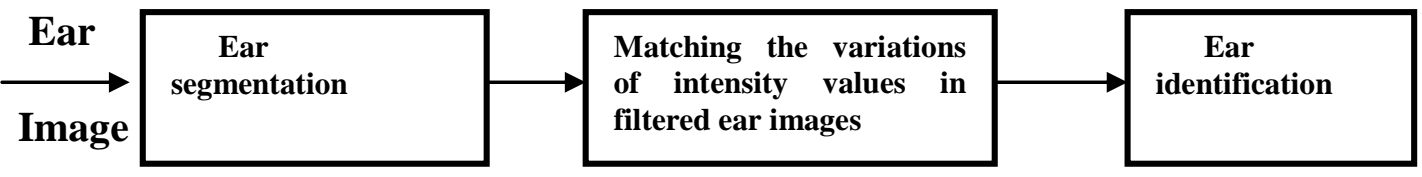

Fig 3.1: Block Diagram

\subsection{Segmenting Ear Image}

For segmenting ear image, first of all it is preprocessed RGB images are converted to grayscale, and then blurred using a $5 \mathrm{X} 5$ Gaussian filter. After blurring the image is then contrast enhanced using the Matlab histeq( ) histogram equalization function and then segment the ear by a three stage ear biometric system [22].

\subsection{Method of Least Squares}

Method of least squares is used to approximate a curve in the graph using best fit straight line such that the sum of error in values given by line is minimized. Formally if there are $\mathrm{n}$ points in the graph with coordinates given by $\left(1, y_{1}\right),\left(2, y_{2}\right), \ldots \ldots \ldots\left(n, y_{n}\right)$ i.e. points are of the form $(i$, $y i)$ where $\mathrm{i}$ is distance of the point from center and $\mathrm{y}_{\mathrm{i}}$ is intensity value of that point and we want to approximate these points with a line $y=m x+c$ such that error is minimized. Error is given by

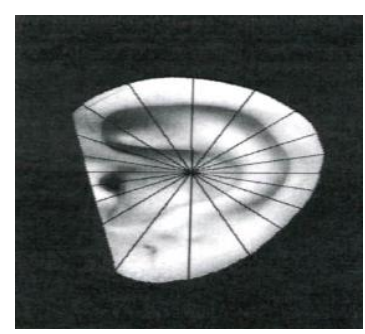

Fig 3.4.1: 20 concurrent lines passing though center of ear are shown, intensity variation along whom is taken for matching the ears.

Now draw the curve between intensity and distance from center for corresponding lines in two different ear images (see Figure 3.4.2.) 


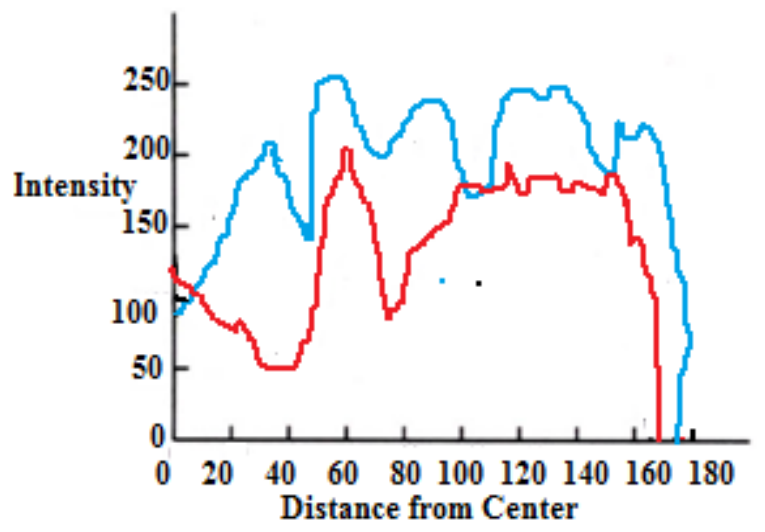

Fig: 3.4.2 Intensity values vs. distance for graph for corresponding lines in two different ear images

Now apply the step 3 of algorithm and approximate the graph shown in fig 3.4.2 and approximated graph is (see Figure 3.4.3.)

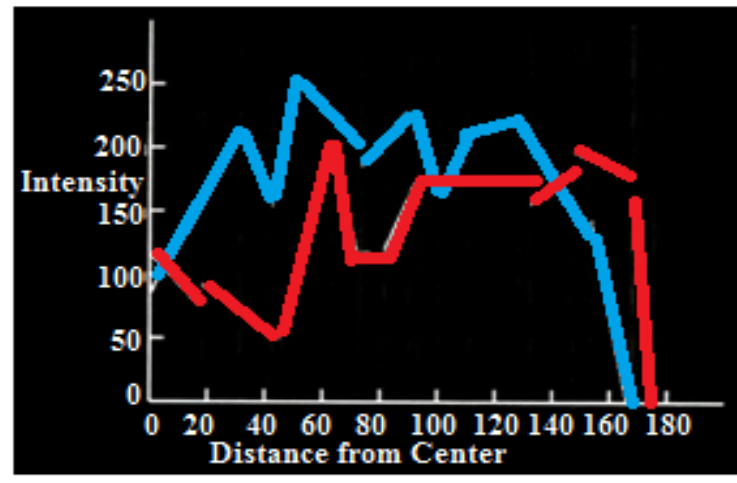

Fig 3.4.3: Approximated graph

Now from the Figure 3.4.3 we implement the step 4 of the algorithm and the fig 3.4.3 shows the various divisions i.e. the region between two vertical lines forms one division. Suppose $r$ is the distance of first point in a division from center and suppose there are s points in one division i.e. distance of points in the division from center is given by $\mathrm{r}, \mathrm{r}+1, \mathrm{r}+2, \ldots \ldots \ldots \ldots \ldots \ldots . \mathrm{r}+\mathrm{s}-1$. Also one approximating line is given by $\mathrm{y}_{1 \mathrm{i}}=\mathrm{m}_{1} \mathrm{x}_{1 \mathrm{i}}+\mathrm{c}_{1}$ and the other line is given by $\mathrm{y}_{2 \mathrm{i}}=\mathrm{m}_{2} \mathrm{x}_{2 \mathrm{i}}+\mathrm{c}_{2}$

Sum of the distances between points for that division is given by

$$
\begin{aligned}
& \text { Distance }_{\text {division }}=\left|\mathrm{y}_{1 \mathrm{i}}-\mathrm{y}_{2 \mathrm{i}}\right| \\
& =\left|\left(\mathrm{m}_{1} \mathrm{x}_{1 \mathrm{i}}+\mathrm{c}_{1}\right)-\left(\mathrm{m}_{2} \mathrm{x}_{2 \mathrm{i}}+\mathrm{c}_{2}\right)\right| \\
& =\mid\left[\mathrm{m}_{1}(\mathrm{r}+\mathrm{r}+1+\mathrm{r}+2+\ldots \mathrm{r}+\mathrm{s}-1)+\mathrm{sc}_{1}\right]- \\
& {\left[\mathrm{m}_{2}(\mathrm{r}+\mathrm{r}+1+\mathrm{r}+2+\ldots \mathrm{r}+\mathrm{s}-1)+\mathrm{sc}_{2}\right] \mid} \\
& \left|\left(\mathrm{m}_{1}-\mathrm{m}_{2}\right)(\mathrm{rs}+\mathrm{s}(\mathrm{s}-1) / 2)+\mathrm{s}\left(\mathrm{c}_{1}-\mathrm{c}_{2}\right)\right|
\end{aligned}
$$

Sum of the distance in all the divisions is taken and further the sum is averaged to get average point wise distance for that line. Now the average value of distance is calculated for all the concurrent lines in two corresponding ears.

Now apply the step 5 of the algorithm i.e. their average gives the matching score.

\section{RESULTS AND DISCUSSIONS}

The experimental setup consists of taking 3 ear images per person. A total of 300 images were taken which belonged to 100 different persons. 20 concurrent lines passing through centroid of ear were taken. Grey level intensity values along each line were approximated by 10 approximating lines.We get the accuracy of $97.67 \%$.

FAR/FRR vs. threshold curve for ear recognition is below (see Figure 4.1) and the ROC curve is (see Figure 4.2)

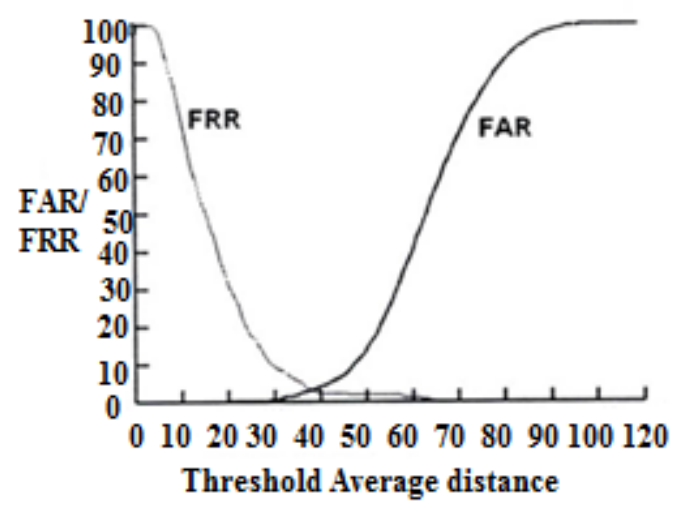

Fig 4.1: FAR/FRR vs. threshold average distance

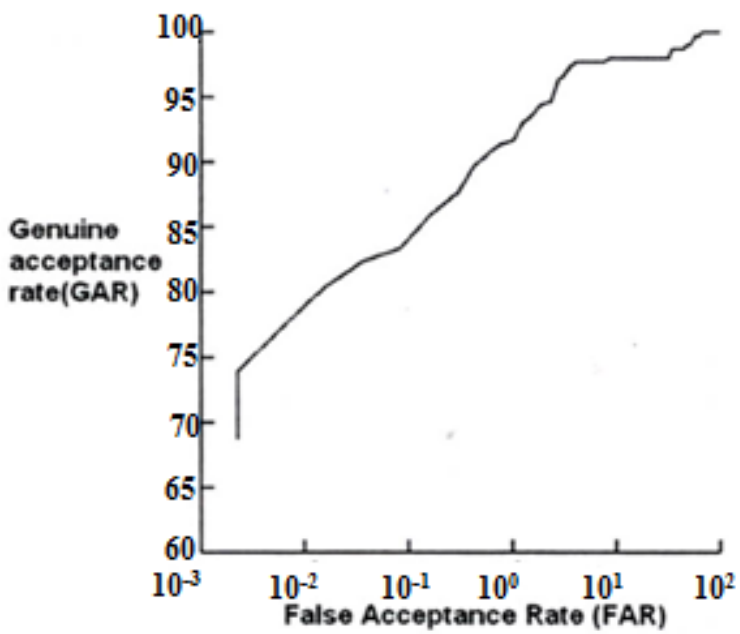

Fig 4.2: ROC Curve

\section{CONCLUSION}

A technique for passive human identification using ears is presented. The approach for dimensionality reduction and feature extraction from 2D ear images is simple and effective. Further the technique for human identification using ears can be improved by combining results from various other representations of ear image which are less effected from illumination changes instead of taking only gray level intensity values. 


\section{REFERENCES}

[1] Bruce E. Wampold, "Estimating Variability in Outcomes Attributable to Therapists: A Naturalistic Study of Outcomes in Managed Care. Journal of Consulting and Clinical Psychology Copyright 2005 by the American Psychological Association 2005, Vol. 73, No. 5, 914-923

[2] Iannarelli Afred, Ear Identification, Forensic Identification Series,Paramount Publishing Company, Fremont, California, 1989.

[3] M. Burge, W. Burger Ear Biometrics, in: Biometrics: Personal Identification in Networked Society (Eds: A.K. Jain, R.Bolle, and S.Pankanti), pp. 273-286, 1998.

[4] D.J. Hurley, M.S. Nixon, J.N. Carter, "Force Field Energy Functionals for Image Feature Extraction," Image and Vision Computing Journal, vol.20, no. 5-6, pp. 311-318, 2002

[5] B. Victor B., K.W. Bowyer, S. Sarkar, "An Evaluation of Face and Ear Biometrics," Proc. of Intl. Conf. on Pattern Recognition, pp. 429-432, 2002.

[6] Moreno, B., Sánchez, Á., Vélez. J.F. On the Use of Outer Ear Images for Personal Identification in Security Applications. IEEE $33^{\text {rd }}$ Annual International Carnahan Conference on Security Technology, 1999, pp. 469-476.

[7] Hurley, D.J.,Nixon,M.S.,and Carter,J.N.(2005). Force Field feature extraction for ear biometrics. Computer Vision and Image Understanding,98(3):491-512.

[8] Bustard, J. D. and Nixon, M. S. (2010). Towards unconstrained ear recognition from two-dimensional images. IEEE Transactions on Systems, Man and Cybernetics A, 40(3):486-494.

[9] Adbel-Mottaleb, M. and Zhou, J. (2006). Human ear recognition from face profile images. In D. Zhang and A. Jain (Eds.), Advances in Biometrics (pp. 786792). Berlin: Springer-Verlag.

[10] Chang, K., Bowyer, K. W., Sarkar, S. and Victor, B. (2003). Comparison and combination of ear and face images in appearance-based biometrics. IEEE Transactions on Pattern Analysis and Machine Intelligence, 25(9):1160-1165.

[11] Moreno, B., Sanchez, A. and Velez, J. F. (1999). On the use of outer ear images for personal identification in security applications. In Proceedings of the IEEE 33rd Annual 1999 International Carnahan Conference on Security Technology (pp. 469-476). Piscataway, NJ: IEEE Press.
[12] Mu, Z., Yuan, L., Xu, Z., Xi, D. and Qi, S. (2004). Shape and structural feature based ear recognition. In $\mathrm{S}$. Li et al. (Eds.), Advances in Biometric Person Authentication (pp. 311-364). Berlin: SpringerVerlag.

[13] Hurley, D. J., Nixon, M. S. and Carter, J. N. (2002). Force field energy functionals for image feature extraction. Image and Vision Computing, 20(5/6):311-317.

[14] Hurley, D. J., Nixon, M. S., and Carter, J. N. (2005). Force field feature extraction for ear biometrics. Computer Vision and Image Understanding, 98(3):491-512.

[15] Choras, M. (2004). Ear biometrics based on geometrical method of feature extraction. In $\mathrm{F}$. Perales and B. Draper (Eds.), Articulated Motion and Deformable Objects (pp. 51-61). Berlin: SpringerVerlag.

[16] Adbel-Mottaleb, M. and Zhou, J. (2006). Human ear recognition from face profile images. In D. Zhang and A. Jain (Eds.), Advances in Biometrics (pp. 786792). Berlin: Springer-Verlag.

[17] Arbab-Zavar, B., Nixon, M. S. and Hurley, D. J. (2007). On model-based analysis of ear biometrics. In Proceedings of the First IEEE International Conference on Biometrics: Theory, Applications, and Systems (pp. 1-5). Piscataway, NJ: IEEE Press.

[18] Chen, H. and Bhanu, B. (2007). Human ear recognition in 3D. IEEE Transactions on Pattern Analysis and Machine Intelligence, 29(4):718-737.

[19] Bhanu, B. and Chen, H. (2003). Human ear recognition in 3D. In Proceedings of the 2003 Workshop on Multimodal User Authentication (pp. 91-98).

[20] Theoharis, T., Passalis, G., Toderici, G. and Kakadiaris, I. (2008). Unified 3D face and ear recognition using wavelets on geometry images. Pattern Recognition, 41(3):796-804.

[21] Yan, P. and Bowyer, K. W. (2007). Biometric recognition using 3D ear shape. IEEE Transactions on Pattern Analysis and Machine Intelligence, 29(8):1297-1308.

[22] Charles Chen(clchen@ colorado.edu) University of Colorado at Boulder November 25,2010. 